\title{
POST-OPERATIVE CONFUSION AND THE PRACTICE OF PEDIATRIC ANESTHESIA
}

Ibrahim Abu-Shahwan MD, Khalid Chowdary MD, Uwe Schwarz MD, Kimmo Murto MD, William Splinter MD. Department of Anesthesiology, Children Hospital of Eastern Ontario, 401 Smyth Rd, University of Ottawa, Ottawa, Ontario, K1H 8L1

INTRODUCTION: With the increasing use of potent and rapid acting inhalation agents, there seem to be an increased incidence of post-op confusion or emergence agitation in children $(1,2)$. There is no single drug that is considered to be the drug of choice for preventing and/or treating this phenomenon $(3,4)$.

METHODS: A standardized questionnaire was sent to 120 members of the Canadian Pediatric Anesthesia Society. Members were given 13 case scenarios involving 5 year old children with different medical condition. Each underwent a different surgery/procedure but emerged with the same post-op behavior. Members were asked to choose from the following treatment options: midazolam, fentanyl, propofol, or one other drug, if any, of their choice to treat post-op confusion.

RESULTS: 51 out of 120 replied. The results of our survey indicate that there is no agreement among pediatric anesthesiologists regarding the treatment of post-op confusion. No single drug was chosen by the majority $(50 \%)$ of pediatric anesthesiologists to be the drug of choice in any of the 13 case scenarios.

\begin{tabular}{|l|l|l|l|l|ll|ll|l|}
\hline \multicolumn{1}{|c|}{ CONDITION } & \multicolumn{2}{|l|}{ midazolam } & \multicolumn{2}{|l|}{ fentanyl } & \multicolumn{2}{l|}{ propofol } & \multicolumn{2}{l|}{ others } \\
\hline ASA I post head MRI for speech delay & 23 & $(47)$ & 3 & $(6)$ & 11 & $(22)$ & 12 & $(24)$ \\
\hline Morbid obesity post MRI for headache & 15 & $(32)$ & 2 & $(4)$ & 15 & $(32)$ & 15 & $(24)$ \\
\hline Ant mediastinal mass post chest MRI & 22 & $(45)$ & 1 & $(2)$ & 11 & $(22)$ & 15 & $(32)$ \\
\hline Autistic post MRI for headache & 8 & $(16)$ & 5 & $(10)$ & 13 & $(27)$ & 23 & $(47)$ \\
\hline Autistic post MRI for headache, premed & 17 & $(35)$ & 0 & $(0)$ & 14 & $(29)$ & 16 & $(33)$ \\
\hline $\begin{array}{l}\text { History of obstructive sleep apnea post T-A } \\
\text { IM codeine 1 mg/kg intra-op }\end{array}$ & 5 & $(10)$ & 19 & $(39)$ & 6 & $(12)$ & 19 & $(39)$ \\
\hline $\begin{array}{l}\text { ASA I post T-A IM codeine 1.5 mg/kg } \\
\text { intra-op }\end{array}$ & 11 & $(22)$ & 19 & $(39)$ & 3 & $(6)$ & 16 & $(33)$ \\
\hline $\begin{array}{l}\text { ASA I post T-A midazolam pre-op IM } \\
\text { codeine 1.5 mg/kg intra-op }\end{array}$ & 5 & $(10)$ & 15 & $(31)$ & 6 & $(12)$ & 23 & $(47)$ \\
\hline $\begin{array}{l}\text { ASA I post inguinal hernia repair, caudal } \\
\text { block, stabile intra-op BP \& HR }\end{array}$ & 12 & $(24)$ & 15 & $(31)$ & 6 & $(12)$ & 16 & $(33)$ \\
\hline $\begin{array}{l}\text { ASA I post hypospadias repair, caudal } \\
\text { epimorph, stabile intra-op BP \& HR }\end{array}$ & 10 & $(21)$ & 17 & $(36)$ & 9 & $(19)$ & 11 & $(23)$ \\
\hline Autistic post dental repair pre-op midazolam & 5 & $(10)$ & 9 & $(18)$ & 9 & $(18)$ & 26 & $(53)$ \\
\hline ASA III, Fontan, post dental repair & 3 & $(10)$ & 11 & $(23)$ & 4 & $(8)$ & 30 & $(63)$ \\
\hline Sickle cell disease, post BMT & 11 & $(22)$ & 11 & $(22)$ & 5 & $(10)$ & 19 & $(39)$ \\
\hline
\end{tabular}

Values displaced are responses $(\%)$

DISCUSSION: Based on the result of our survey, further studies are needed to improve the diagnosis, prevention, and treatment of post-op confusion.

\section{References:}

1-Anesthesiology 1997; 87: 1298-300 2- Anesth Analg 2003; 96: 1625-30

3- Paed Anesth 2003; 13: 609-613 4- Anesthesiology 2004; 100: 1138-45 